\title{
E-Beam lithography designed substrates for surface enhanced Raman spectroscopy ${ }^{\text {is }}$
}

\author{
Neval A. Cinel ${ }^{\mathrm{a}, *}$, Semih Cakmakyapan ${ }^{\mathrm{a}, \mathrm{b}}$, Serkan Butun ${ }^{\mathrm{c}}$, Gulay Ertas ${ }^{\mathrm{d}}$, \\ Ekmel Ozbay a,b,e \\ ${ }^{a}$ Bilkent University, Nanotechnology Research Center, 06800, Ankara, Turkey \\ ${ }^{\mathrm{b}}$ Bilkent University, Physics Department, 06800, Ankara, Turkey \\ ${ }^{\mathrm{c}}$ Technological Institute, Electrical Engineering and Computer Science Department, Northwestern University, Evanston, IL 60208, USA \\ ${ }^{\mathrm{d}}$ METU, Chemistry Department, 06800, Ankara, Turkey

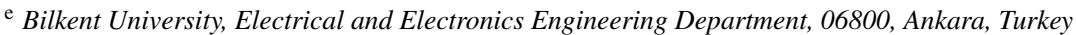

Received 29 September 2014; received in revised form 24 October 2014; accepted 24 November 2014

Available online 25 January 2015

\begin{abstract}
Surface Enhanced Raman Spectroscopy (SERS) is a popular method that amplifies weak Raman signals from Raman-active analyte molecules making use of certain specially-prepared metallic surfaces. The main challenge in SERS is to design and fabricate highly repeatable, predictable, and sensitive substrates. There are many fabrication methods that strive to achieve this goal, which are briefly summarized in this paper. The E-beam lithography method is proposed to be superior to the mentioned techniques. In this paper, we review how EBL can be utilized in the preparation of SERS substrates and we discuss the contributions to the field by the Özbay group.
\end{abstract}

(c) 2015 Elsevier B.V. All rights reserved.

Keywords: Electron beam lithography; Surface enhanced Raman spectroscopy; Nano-cone; Nano-ring; Bowtie; Fractal

\section{Introduction}

SERS has been extensively used as a powerful method for the sensitive and specific detection of a wide range of bio-molecules since it was discovered in $1977[1,2]$. SERS depends on a phenomenon called Raman scattering. When a photon interacts with an atom or molecule, a very small percentage of the photons are scattered with a frequency less than the incoming photons. The energy

\footnotetext{
The article was accepted from PECS - XI conference.

* Corresponding author.

E-mail address: nyilmaz@ee.bilkent.edu.tr (N.A. Cinel).
}

difference is dissipated at the vibrational modes of the molecule in interaction. If the scattered light intensity is plotted against frequency, the Raman spectrum of the molecule can be obtained. Every chemical bond in the molecule stands for a different peak in the spectrum. The Raman spectra are just like the finger print of the molecule in this manner. This phenomenon is enhanced at metallic, nano-structured surfaces by $10^{5}-10^{6}$ and is named SERS.

Electromagnetic (EM) and chemical mechanisms are considered as the factors that enable surface enhanced Raman scattering [3,4]. The dominant mechanisms among the two are the EM mechanism which is due to the excitation of the localized surface plasmons (LSP) on nano-roughened surfaces. The LSP is the collective 
oscillation of the surface electrons that are excited by the electromagnetic radiation that interacts with the nanosized metallic particles. The frequency and strength of this oscillation depends on the type of the metal and dielectric environment, the size, shape, and size distribution of the nanoparticles. The optimum signal intensity is obtained when the laser frequency is selected to match the LSP resonance of the nano-structured surface. Due to this, to enhance the signal intensity of SER scattering, all the factors that affect LSP resonance should be controlled.

After the discovery of SERS, several fabrication techniques have been developed to attain stabilized substrates that have long shelf lives and high quality. The first SERS-active surfaces were obtained by chemical methods such as oxidation-reduction cycling [5], electro-chemical roughening [6] and island lithography [7]. In oxidation-reduction cycling, repeated oxidationreduction cycles are applied to the metallic $(\mathrm{Ag})$ surfaces ending up with roughened surfaces at the nano-scale. In electro-chemical roughening the metallic surfaces are immersed in highly concentrated acidic solutions for brief periods to chemically roughen the surfaces. The metal island lithography method, takes its name from the island like distribution of the $\mathrm{Ag}$ films evaporated onto a proper substrate. In all the mentioned techniques, unreproducible and heterogeneous substrates that have a large surface area but low SERS enhancement are obtained.

There are also lithographic techniques such as nanosphere lithography [8], oblique angle deposition [9]. In nanosphere lithography, the SERS-active substrates are obtained by coating a self-assembled monolayer of polystyrene nanoparticles with metal evaporation. In oblique angle deposition, the sample is held at an angle during metal evaporation. Cylindrical, irregular shaped and randomly distributed nano-rod arrays are obtained due to the surface diffusion and shadowing effect [10]. Other commonly used methods are to directly use colloidal metallic solutions or using them fixed on a substrate [11]. The colloidal solutions are obtained by the reduction of diluted metallic salt solutions. In this method, the differences in metallic particles sizes and their shapes due to the differences in preparation recipes can lead to several orders of magnitude difference in the SERS enhancement factor.

Electron Beam Lithography overcomes all the aforementioned difficulties. The substrates have high repeatability and stability. They can be designed to work at desired wavelengths and the SERS enhancement factor can be calculated with the help of simulations to save manpower and money. The verification of the designs can be done by optical transmission measurements easily. The method has some weaknesses, too. The time that is elapsed for the pixel by pixel scan to be complete can be long in addition to low throughput. The system maintenance can be expensive and tedious. Metal lift-off and etching can be troublesome for nanoscale features. In spite of these difficulties, the reproducibility and predictability of the results, which is the key aspect in SERS substrate preparation, make EBL an ideal candidate for fabrication [12].

The best metric used in the comparison of the SERSactive surfaces is the SERS enhancement factor. The most commonly used enhancement factor calculation can be written as $\mathrm{EF}=\left(I_{\mathrm{SER}} / N_{\text {surf }}\right) /\left(I_{\mathrm{R}} / N_{\mathrm{vol}}\right)[13-15]$. Here $N_{\mathrm{Vol}}$ is the number of molecules in the detection volume that contributes to the Raman signal, $N_{\text {surf }}$ is the number of molecules attached on the surface that contribute to the SER signal. $I_{\mathrm{R}}$ and $I_{\mathrm{SER}}$ are the intensities of the Raman and SER signals. The molecules with known packing densities are preferentially used in these calculations. The best known molecule used for this aim is Benzenethiol. Two measurements, one from a known detection volume of liquid benzenethiol and the other from benzenethiol applied on the nano-structured surface are taken and the mentioned formula is used for the calculation of the SERS enhancement factor. This enables an objective comparison to be made between two lithographic surfaces. Such a comparison is not clear for surfaces prepared with other methods since the total metallic area and, therefore, $N_{\text {surf }}$ cannot be determined accurately.

In the literature, there are many SERS-active surface designs prepared with EBL [12,16-18]. These designs are mainly periodic arrays of simple nano-structures and generally the relation between the LSP resonance wavelength and SER signal enhancement is studied. To give several examples, Le Ru, has taken SERS measurements, from periodic gold dot, square and triangle arrays from "Rhodamine 6G" [19]. In a very similar study, Gunnarsson, studied similar Ag structures on silicon wafer for the same molecule and reported that better results are obtained when compared with nano-roughened Ag film [20]. Kahl, has shown that the SERS measurements of "Rhodamine $6 \mathrm{G}$ " on gold periodic nano-dot arrays and grating structures resulted in an order of magnitude better SERS enhancement when compared with metalisland film substrates [21]. Finally, Hatap, showed that an SERS enhancement of $10^{11}$ has been obtained from free-standing gold bowtie nano-antennas [22]. The diversity of designs is endless when fabrication with EBL is considered. Below, we summarize the contributions to substrate design with EBL for SERS by Özbay group, 
a

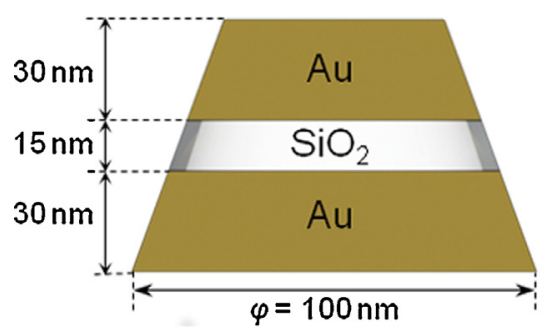

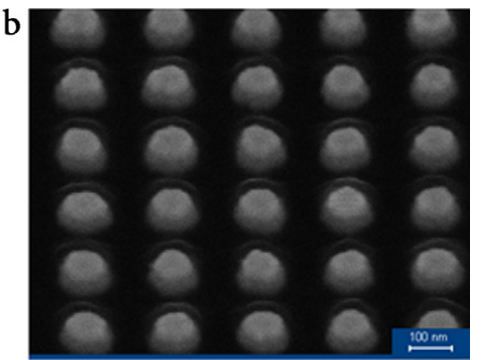

Fig. 1. (a) physical dimensions of a single nano-cone (b) SEM image of an array of nano-cones with period of $200 \mathrm{~nm}$ and a base diameter of $100 \mathrm{~nm}$.

namely tandem structures, concentric rings, and fractal bowtie antennas.

\section{Tandem structures}

In this study, periodic $\mathrm{Au}-\mathrm{SiO}_{2}$ - $\mathrm{Au}$ Nano-cone arrays are fabricated with EBL on sapphire substrate and are shown to provide a 10 times higher SER signal intensity when compared with periodic Au nano-cone arrays fabricated with the same method [23]. Au nano-cone arrays provide single resonance tuned to the laser wavelength. On the other hand, $\mathrm{Au}-\mathrm{SiO}_{2}-\mathrm{Au}$ nano-cone arrays as shown in Fig. 1 can provide double resonance tuned to the laser and the Stokes wavelength, respectively. The high tunability of the $\mathrm{Au}-\mathrm{SiO}_{2}$-Au nano-cone arrays comes from the used fabrication method. The nanodots written by EBL were then coated with gold and $\mathrm{SiO}_{2}$ by electron beam evaporation method. The details of the fabrication are described in previous reports and, therefore, not repeated here [23]. Several different base diameter nano-cones were fabricated with $5 \mathrm{~nm}$ increments in a base diameter successfully that enabled tuning the resonance behavior of the substrate in addition to the metal heights which can be varied easily with the E-Beam evaporation method. Such highly tunable and predictable substrates are impossible to obtain with other techniques described earlier in this report.

Since EBL is a highly precise method in which the intended and fabricated structures show a good match in shape and size, their resonance behavior can be predicted by simulations. In this study, the double resonance behavior of the nano-cones was first shown by simulations and then verified by optical measurements. By this way, one can have a good estimate of the resonance behavior of the intended nanostructures ahead of fabrication, and thereby reduce the fabrication costs and time. The simulations are done by Lumerical software that uses the Finite Difference Time Domain (FDTD) method. The simulation and measurement results are shown in Fig. 2 for nanocones that have a bottom radius of $55 \mathrm{~nm}$. The broadening and intensity of the peaks differ due to the discrepancies in shape and size of the nanoparticles fabricated and simulated. However, the positions of the resonance dips are in good correlation.

The SERS enhancement factor can also be predicted by simulations for EBL fabricated structures via the use of 3D electric field monitors. The electric field distribution obtained at excitation and Stokes shifted wavelengths are used to calculate $\Sigma\left|E_{\text {exc }}\right|^{2} .\left|E_{\text {stokes }}\right|^{2}$ over the exposed volume. The results are then normalized with respect to the total surface area. In this study, the results obtained for "tandem" structures are compared with "only gold" structures to make a guess ahead of SERS experiments. The resultant enhancement is approx. 12 for a $55 \mathrm{~nm}$ radius array for the Raman line at $1575 \mathrm{~cm}^{-1}$. The experimental values obtained for the same conditions are approx. 9 for a $55 \mathrm{~nm}$ radius array which is in good accordance with the simulations.

The comparison of SER signal intensity for an array of tandem nano-cones with a period of $200 \mathrm{~nm}$ and a

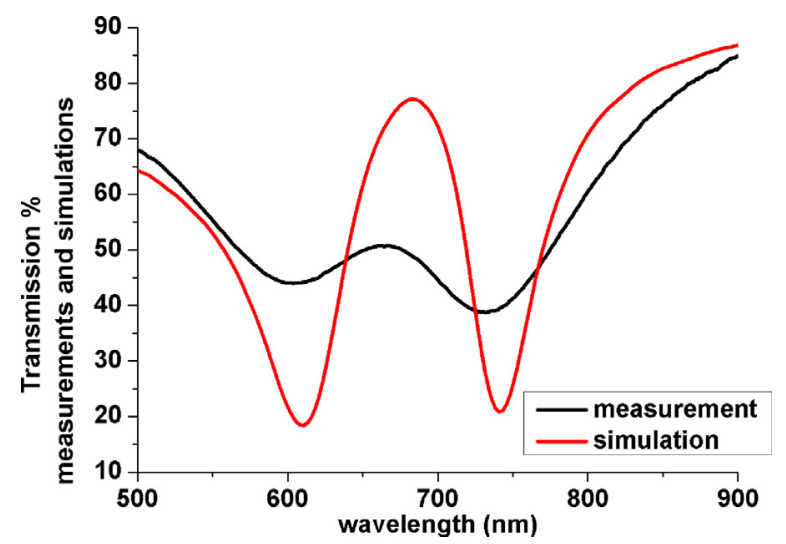

Fig. 2. Comparison of the transmission and simulation results for an array of nano-cones with a period of $200 \mathrm{~nm}$ and a base diameter of $110 \mathrm{~nm}$. 


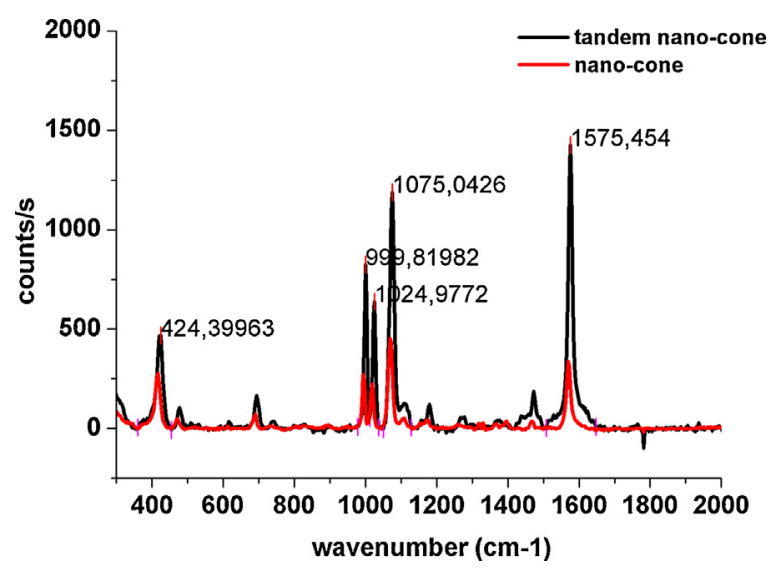

Fig. 3. Comparison of SER signal intensity for an array of tandem nano-cones with a period of $200 \mathrm{~nm}$ and a base diameter of $110 \mathrm{~nm}$ and its single resonance counterpart. The excitation wavelength is $632.8 \mathrm{~nm}$.

base diameter of $110 \mathrm{~nm}$ and its single resonance counterpart are shown in Fig. 3. The SERS enhancement predicted through simulations and measured via SERS experiments have shown that the tandem nano-cone design provided 10 times higher SER signal intensity when compared with periodic Au nano-cone arrays fabricated with the same method. The enhancement would be more prominent if the comparison was made with respect to simply nano-roughened metallic surfaces, as shown in another study conducted by the Ozbay group [24].

\section{Concentric ring structures}

Concentric arcs and rings are used for various purposes in the literature mainly for their focusing properties [25] or as plasmonic lenses [26]. In our study, circular concentric rings etched from a planar gold film are compared with their coupled counterparts and plain gold film in terms of their SERS performance [24]. In the coupled design, the etched parts are filled with a dielectric and then layered up with gold circular concentric rings as shown in the inset of Fig. 4(b).

In this study, it can be seen that EBL can be used to obtain structures both indented and protruding. To etch the rings from plain gold a negative tone resist were used in the e-beam lithography and then an $\mathrm{O}_{2}$ plasma etch was conducted. The details of the fabrication steps are described elsewhere [24].

Fig. 4 depicts the SER spectrum of Benzenethiol from the "coupled" rings and the "etched" rings. Here, the etched ring design is optimized to maximize the focusing property by choosing its period to be equal to the surface plasmon wavelength. The coupled ring design is fabricated with the same physical dimensions for better comparison. The SERS measurements show that the coupled ring design can provide 630 times better enhancement than plain gold film and 8 times better enhancement than the etched film. This is attributed to the increased number of "hotspots" due to the coupling between upper and lower rings. The enhancement factor calculated as described in the introduction is $1.67 \times 10^{7}$ for the coupled-concentric ring structures. The SERS enhancement factors experimentally obtained are compared with those calculated by FDTD simulations where the SERS intensity is taken to be proportional to the fourth power of E-field distribution at the laser excitation frequency. The ratio $|\mathrm{E}|^{4}$ coupled $/|\mathrm{E}|^{4}$ etched is 12 for the simulations which is in accordance with the experimental ratio, eight times larger signal intensity.

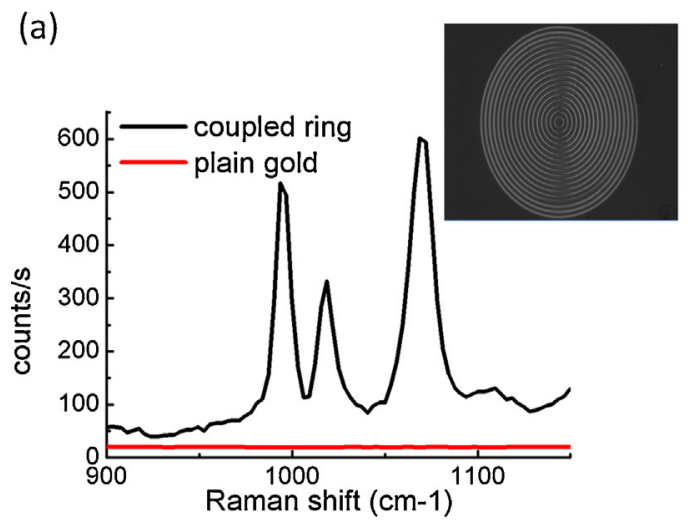

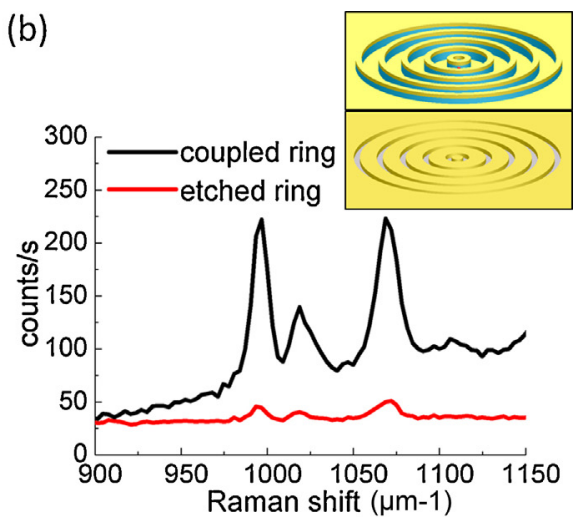

Fig. 4. (a) SERS measurements taken from coupled ring and plain gold (inset: SEM figure taken for coupled ring structure, period of the rings: $500 \mathrm{~nm}$, inner ring diameter: $975 \mathrm{~nm}$ ). (b) SERS measurements taken from coupled ring and plain gold. Period of the rings is approximately $615 \mathrm{~nm}$ and the diameter of the center disc is around $1.45 \mu \mathrm{m}$. (Inset: conceptual figures of coupled ring and etched ring structures) The excitation wavelength used in SERS measurements is $632.8 \mathrm{~nm}$. 
(a)

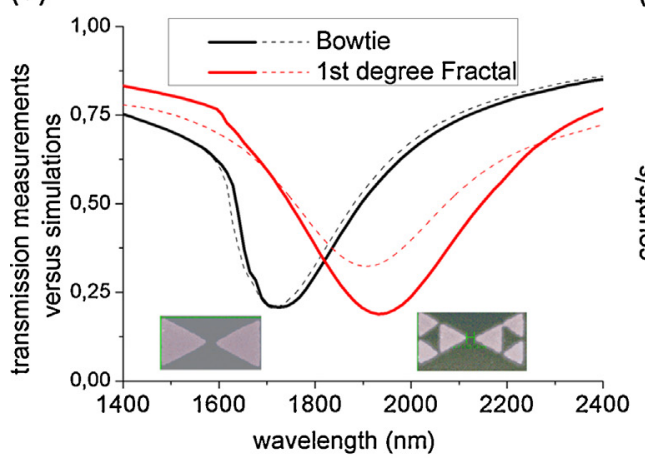

(b)

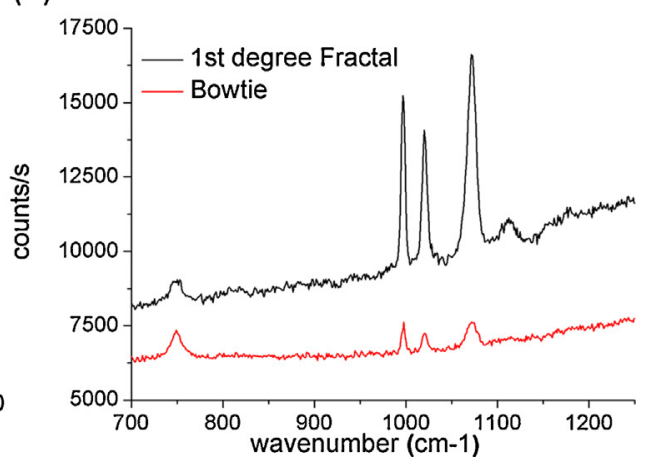

Fig. 5. (a) Transmission measurements versus simulations (inset: SEM figures of the fabricated structures.) (b) SERS measurements for bowtie nano-antennas and their first degree counterparts ( $g a p=65 \mathrm{~nm}$, sides of triangles $\sim 400 \mathrm{~nm}$ for both figures). The excitation wavelength used in SERS measurements is $785 \mathrm{~nm}$.

\section{Bowtie and fractal bowtie structures}

Bowtie structures offer a wide diversity of tuning mechanisms for enhanced E-field generation at desired wavelengths due to the excitation of localized surface plasmons. In bowtie structures, the LSP resonance can be tuned by changing the spacing between the two triangle prisms [22,27], the triangle angles [28,29] and sizes [30] to obtain better SERS enhancement factors. EBL, offers advantages such as decreasing the spacing down to $5 \mathrm{~nm} \mathrm{[31]} \mathrm{and} \mathrm{sensitively} \mathrm{tuning} \mathrm{the} \mathrm{physical}$ parameters. Obtaining fractal structures is even possible.
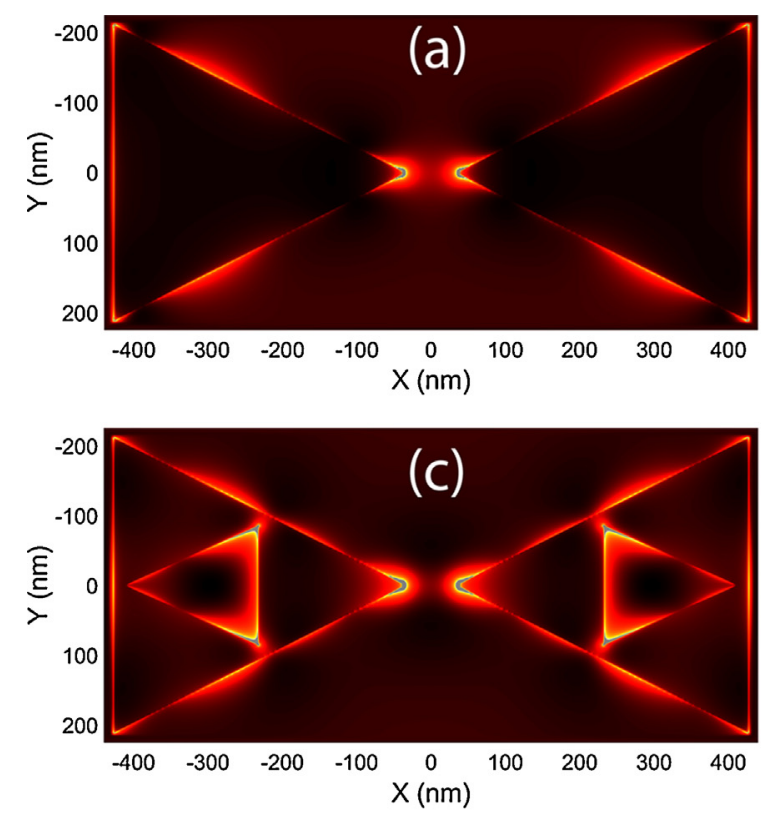

0

5

10
To our knowledge, the Özbay group recently reported the fabrication of first and second degree fractal bowtie nano-antennas for the first time [32].

The design of the bowtie and fractal structures were done by FDTD simulations and verified by transmission measurements. The calculated and measured transmission spectra of the structures are in good accordance as shown in Fig. 5(a). The resonance wavelength of the structures are in NIR, which enable them to be used in NIR-SERS applications, which are preferred especially to overcome the disadvantages of visible lasers such as photochemical reactions, background from
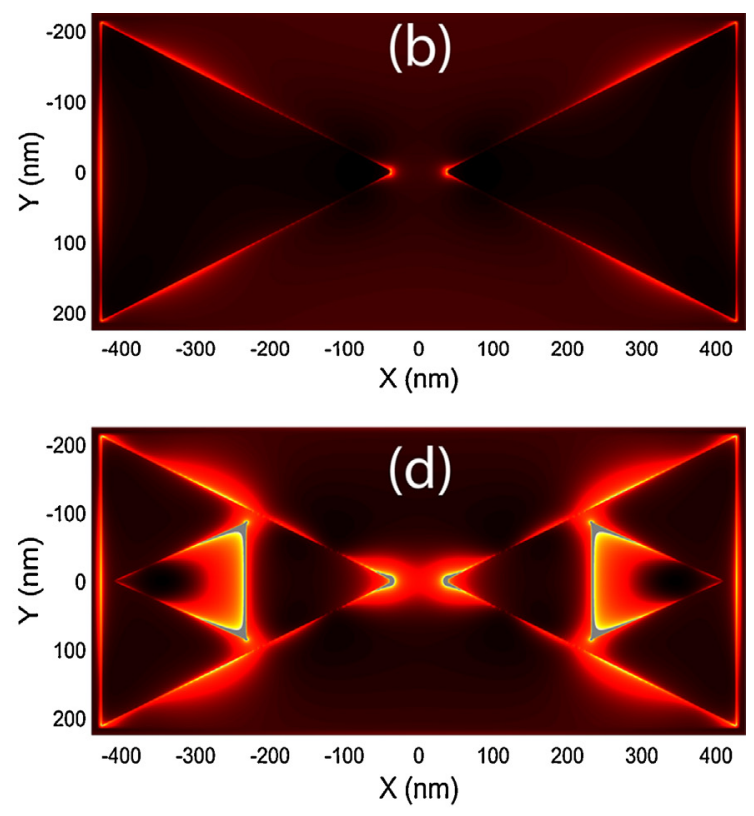

15

Fig. 6. Electric field distributions at the laser (a, c) and Stokes shifted wavelength (b, d) $785 \mathrm{~nm}$ and $895 \mathrm{~nm}$, respectively. 
fluorescence, and degeneration of molecules [33,34]. The SERS measurements taken from bowtie and fractal nanoantennas were compared and the SERS signal intensity was reported to be increased for the first degree fractals. Raman measurements of the structures depict that SERS signal intensity at $1075 \mathrm{~cm}^{-1}$ is enhanced by a factor of eight for the first degree fractal when compared to the bowtie nano-antenna as shown in Fig. 5(b).

The electric field distribution is also obtained through simulations at both the excitation and Stokes wavelengths as shown in Fig. 6. The intensified E-field at both wavelengths at the tip of the triangles and the gaps lead to appreciable SERS enhancement. The increase in SERS signal intensity for fractal nano-antennas is attributed to the generated hotspots in the cavities of the fractal structure as a result of the plasmonic coupling between the subwavelength features. The SERS enhancement factor is estimated by calculating $\Sigma\left|E_{\text {exc }}\right|^{2} .\left|E_{\text {stokes }}\right|^{2}$ over the gold areas. The simulations showed that the integrated Efield is 24 times larger for the first degree fractal structure when compared with the bowtie design.

\section{Summary}

The high sensitivity and tunability obtained with EBL can be used to reduce the time, effort, and cost in the design phase of the SERS substrates with the help of simulations. Through simulations, the physical dimensions required to obtain the desired resonance wavelengths can be studied before the fabrication starts. The SERS enhancement can even be predicted through calculations using electric field distributions observed by 3D monitors. This approach can be applied to a variety of geometries such as nano-cones, concentric rings and bowtie nano-antennas.

\section{Acknowledgements}

This work is supported by the projects DPT-HAMIT, DPT-FOTON, and NATO-SET-193 and TUBITAK under the project nos. 113E331, 109A015 and 109E301. One of the authors (E.O.) also acknowledges partial support from the Turkish Academy of Sciences.

\section{References}

[1] D.L. Jeanmaire, R.P. Van Duyne, Surface Raman spectroelectrochemistry: part I. Heterocyclic, aromatic, and aliphatic amines adsorbed on the anodized silver electrode, J. Electroanal. Chem. Interfacial Electrochem. 84 (1977) 1-20.

[2] M.G. Albrecht, J.A. Creighton, Anomalously intense Raman spectra of pyridine at a silver electrode, J. Am. Chem. Soc. 99 (1977) 5215-5217.
[3] C.L. Haynes, A.D. McFarland, R.P. Van Duyne, Surfaceenhanced Raman spectroscopy, Anal. Chem. 77 (2005) 338a-346a (Sep 1).

[4] K. Kneipp, M. Moskovits, H. Kneipp, Surface-Enhanced Raman Scattering: Physics and Applications, first ed., Springer, Berlin, 2006.

[5] M. Fleischmann, P. Hendra, A. McQuillan, Raman spectra of pyridine adsorbed at a silver electrode, Chem. Phys. Lett. 26 (1974) 163-166.

[6] A. Kudelski, Raman studies of rhodamine 6G and crystal violet sub-monolayers on electrochemically roughened silver substrates: do dye molecules adsorb preferentially on highly SERS-active sites? Chem. Phys. Lett. 414 (2005) 271-275 (Oct 14).

[7] M. Green, F.M. Liu, SERS substrates fabricated by island lithography: the silver/pyridine system, J. Phys. Chem. B 107 (2003) 13015-13021 (Nov 27).

[8] C.L. Haynes, R.P. Van Duyne, Nanosphere lithography: a versatile nanofabrication tool for studies of size-dependent nanoparticle optics, J. Phys. Chem. B 105 (2001) 5599-5611 (Jun 21).

[9] S.B. Chaney, S. Shanmukh, R.A. Dluhy, Y.-P. Zhao, Aligned silver nanorod arrays produce high sensitivity surface-enhanced Raman spectroscopy substrates, Appl. Phys. Lett. 87 (2005) 031908.

[10] T. Karabacak, G.-C. Wang, T.-M. Lu, Physical self-assembly and the nucleation of three-dimensional nanostructures by oblique angle deposition, J. Vacuum Sci. Technol. A 22 (2004) 1778-1784.

[11] R.G. Freeman, K.C. Grabar, K.J. Allison, R.M. Bright, J.A. Davis, A.P. Guthrie, et al., Self-assembled metal colloid monolayers: an approach to SERS substrates, Science 267 (1995) $1629-1632$.

[12] M. Kahl, E. Voges, S. Kostrewa, C. Viets, W. Hill, Periodically structured metallic substrates for SERS, Sens. Actuators B-Chem. 51 (1998) 285-291 (Aug 31)

[13] E.C. Le Ru, E. Blackie, M. Meyer, P.G. Etchegoin, Surface enhanced Raman scattering enhancement factors: a comprehensive study, J. Phys. Chem. C 111 (2007) 13794-13803 (Sep 20).

[14] C.L. Haynes, R.P. Van Duyne, Plasmon-sampled surfaceenhanced Raman excitation spectroscopy, J. Phys. Chem. B 107 (2003) 7426-7433 (Jul 31).

[15] A.D. McFarland, M.A. Young, J.A. Dieringer, R.P. Van Duyne, Wavelength-scanned surface-enhanced Raman excitation spectroscopy, J. Phys. Chem. B 109 (2005) 11279-11285 (Jun 9).

[16] J. Beermann, S.M. Novikov, K. Leosson, S.I. Bozhevolnyi, Surface enhanced Raman imaging: periodic arrays and individual metal nanoparticles, Opt. Express 17 (2009) 12698-12705 (Jul 20).

[17] N. Guillot, H. Shen, B. Fremaux, O. Peron, E. Rinnert, T. Toury, et al., Surface enhanced Raman scattering optimization of gold nanocylinder arrays: influence of the localized surface plasmon resonance and excitation wavelength, Appl. Phys. Lett. 97 (2010) (Jul 12).

[18] J.M. Oran, R.J. Hinde, N. Abu Hatab, S.T. Retterer, M.J. Sepaniak, Nanofabricated periodic arrays of silver elliptical discs as SERS substrates, J. Raman Spectrosc. 39 (2008) 1811-1820 (Dec).

[19] E. Le Ru, P. Etchegoin, J. Grand, N. Félidj, J. Aubard, G. Lévi, et al., Surface enhanced Raman spectroscopy on nanolithographyprepared substrates, Curr. Appl. Phys. 8 (2008) 467-470

[20] L. Gunnarsson, S. Petronis, B. Kasemo, H. Xu, J. Bjerneld, M. Käll, Optimizing nanofabricated substrates for surface enhanced Raman scattering, Nanostr. Mat. 12 (1999) 783-788. 
[21] M. Kahl, E. Voges, S. Kostrewa, C. Viets, W. Hill, Periodically structured metallic substrates for SERS, Sens. Actuators B: Chem. 51 (1998) 285-291.

[22] N.A. Hatab, C.-H. Hsueh, A.L. Gaddis, S.T. Retterer, J.-H. Li, G. Eres, et al., Free-standing optical gold bowtie nanoantenna with variable gap size for enhanced Raman spectroscopy, Nano Lett. 10 (2010) 4952-4955.

[23] N.A. Cinel, S. Butun, G. Ertas, E. Ozbay, Fairy chimney'-shaped tandem metamaterials as double resonance SERS substrates, Small 9 (Feb 25 2013) 531-537.

[24] N.A. Cinel, S. Cakmakyapan, G. Ertas, E. Ozbay, Concentric ring structures as efficient SERS substrates, IEEE J. Sel. Top. Quantum Electron. 19 (2013) 4601605 (May-Jun).

[25] J.M. Steele, Z. Liu, Y. Wang, X. Zhang, Resonant and nonresonant generation and focusing of surface plasmons with circular gratings, Opt. Express 14 (2006) 5664-5670.

[26] W.B. Chen, D.C. Abeysinghe, R.L. Nelson, Q.W. Zhan, Plasmonic lens made of multiple concentric metallic rings under radially polarized illumination, Nano Lett. 9 (2009) 4320-4325 (Dec).

[27] D.P. Fromm, A. Sundaramurthy, P.J. Schuck, G. Kino, W. Moerner, Gap-dependent optical coupling of single bowtie nanoantennas resonant in the visible, Nano Lett. 4 (2004) 957-961.
[28] W. Ding, R. Bachelot, S. Kostcheev, P. Royer, R.E. de Lamaestre, Surface plasmon resonances in silver bowtie nanoantennas with varied bow angles, J. Appl. Phys. 108 (2010) 124314.

[29] G. Bi, L. Wang, L. Ling, Y. Yokota, Y. Nishijima, K. Ueno, et al., Optical properties of gold nano-bowtie structures, Opt. Commun. 294 (2013) 213-217.

[30] L. Wang, X. Xu, Spectral resonance of nanoscale bowtie apertures in visible wavelength, Appl. Phys. A 89 (2007) 293-297.

[31] R. Murali, E. Walters, F. Zaman, C. Tabor, W. Huang, M. ElSayed, et al., Fabrication of bow-tie nano-gap structures by electron beam lithography, in: Nano Science and Technology Institute (NSTI) Nanotechnology Conference and Trade Show Technical Proceedings, 2007, 258-260.

[32] S. Cakmakyapan, N.A. Cinel, A.O. Cakmak, E. Ozbay, Validation of electromagnetic field enhancement in near-infrared through Sierpinski fractal nanoantennas, Opt. Express 22 (2014) 19504-19512.

[33] S. Mahajan, M. Abdelsalam, Y. Suguwara, S. Cintra, A. Russell, J. Baumberg, et al., Tuning plasmons on nano-structured substrates for NIR-SERS, Phys. Chem. Chem. Phys. 9 (2007) 104-109.

[34] K. Kneipp, R.R. Dasari, Y. Wang, Near-infrared surface-enhanced Raman scattering (NIR SERS) on colloidal silver and gold, Appl. Spectrosc. 48 (1994) 951-955. 